\title{
COMMUNICABLE DISEASES REPORT, NSW, FOR MARCH AND APRIL 2004
}

For information on communicable diseases in New South Wales that is updated regularly, visit the website www.health.nsw.gov.au and click on Infectious Diseases.

\section{TRENDS}

Tables 2 and 3 and Figure 1 show reports of communicable disease received through to the end of April 2004 in NSW.

Notifications of arboviruses peaked in March, most of which were due to infection with Ross River virus, mainly from the rural north of the state. Notifications of pertussis continued to decline through autumn, after peaking in November 2003. No cases of measles were reported in April in NSW.

To the end of April, 40 cases of meningococcal disease have been reported in NSW, including one death (due to serogroup $\mathrm{C}$ disease). For the same period last year, 45 cases were reported, including one death.

Notifications of cryptosporidiosis are slowly declining after peaking in January. While no single source of infection has been identified, some cases may have been acquired from close contact with other infectious cases (including cases at childcare settings) and from swimming in contaminated swimming pools. Guidelines for the prevention of cryptosporidiosis in these settings are available from your local public health unit or from www.health.nsw.gov.au/public-health/ehb/general/ pools/publicpools.html.

\section{TWO CLUSTERS OF MEASLES LINKEDTO OVERSEASTRAVEL}

In March, a mother and young child who reside in the Northern Rivers Area Health Service returned to Australia after acquiring measles while travelling in India. Their infection was investigated by staff from the Northern Rivers Public Health Unit (NRPHU), who assessed the child to have been in the highly infectious phase while returning home. The mother reported that she and the child had never received measles vaccine. Contacts at risk of infection in Australia included susceptible people who shared two flights to Brisbane, others at the airport, and the other patients and staff at the medical clinic attended by the child. In response, NSW Health issued a media release warning other travellers to be alert for signs of measles, and the airline company agreed to contact passengers on the flight to alert them of the risk of infection. The NRPHU traced other social and health care contacts of the cases and offered immunisation or immunoglobulin to those susceptible to infection.

Later in the month, South Eastern Sydney Public Health Unit (SESPHU) investigated the case of a young man who developed measles after returning from Japan. Before the onset of his rash, he attended a concert at a local club. The SESPHU traced close contacts at risk of infection, and issued local alerts to other patrons through the media and a sign at the club. Two weeks later, three secondary cases in young adults who had attended the same concert were reported by clinicians. The SESPHU found that these subsequent cases in turn had had large numbers of contacts while infectious, including guests at a wedding, participants in a multi-day bike race, work and social contacts, and other patients sharing medical waiting rooms. Large numbers of people were also potentially exposed via public transport and other public venues. A general media alert was issued.

Due mainly to high levels of immunisation in NSW, measles is now uncommon but intermittent outbreaks associated with overseas travellers still occur. Measles is highly infectious via airborne droplets and can be acquired just by being in the same room with an infected person. Measles infection can lead to serious complications including pneumonia and encephalitis.

Measles may one day be eliminated through high levels of community immunisation. While great strides have been made in recent years in improving the rates of childhood immunisation, these incidents highlight the need to target people aged in their 20s and 30s, overseas travellers, and health care workers, for measles vaccination. All children should be routinely vaccinated against measles at ages 12 months and 4 years. Everyone else born in or after 1966, without documentation of immunity, should have had two doses of measles vaccine. Patients suspected of having possible measles infection should be rapidly isolated from other patients.

\section{TWO CLUSTERS OF LEGIONNAIRES' DISEASE}

In March, two clusters of Legionnaires' disease were identified, and here we report on the characteristics of the clusters and the resulting public health actions.

Legionnaires' disease is a form of pneumonia, caused by infection with various species of Legionella bacteria. In NSW, the species L. pneumophila is the most common cause of Legionnaires' disease, followed by $L$. longbeachae. Symptoms of the disease include: fever, chills, cough, muscle aches, headache, tiredness, loss of appetite, and diarrhoea. People who have underlying disease, or are older, or who smoke, are more susceptible to infection. The mortality rate is around $10-15$ per cent. The incubation period varies from $2-10$ days and is typically 5-6 days. The disease is not spread from person to person. 


\section{Legionella pneumophila infection}

In March 2004, investigations by the South Eastern Sydney Public Health Unit (SESPHU) identified a possible linked cluster of six cases of Legionnaires' disease caused by L. pneumophila.

The onset of illness for these people ranged from late January to early March. They were aged between 23 and 65 years and four were male. Five reported movements in one small area of Oxford Street during their possible exposure period. Four had also visited various other areas of the Central Business District (CBD) of Sydney. Another person with Legionnaires' disease due to Legionella pneumophila who was hospitalised in the Hunter Area Health Service was also found to have stayed in the Sydney CBD for two days in this period.

L. pneumophila is the most common cause of Legionnaires' disease in NSW (55 per cent of reported cases between 1991-2000, or between 16 and 80 cases per year). L. pneumophila infections have been associated with inhalation of aerolised water containing the organism, often from contaminated cooling towers or occasionally domestic water supplies and spa pools.

The SESPHU alerted local hospital and general practitioners, and worked with the City of Sydney Council to assess the routine disinfection processes of nearby cooling towers and of a large fountain. No source of the outbreak was identified. The City of Sydney Council wrote to the operators of the approximately 1,800 cooling towers in the CBD to reinforce the need for careful assessment and disinfection of cooling towers. To identify any further related cases, all NSW public health units were advised of the cluster and requested to contact their local hospitals and laboratories for other possible cases of Legionnaires' disease and question any suspected cases about their movements in the 10 days before onset of illness. NSW Health issued a statewide media release to alert the public and building operators.

By the end of March a further two, possibly linked, cases were identified, both with onset of illness in early March. There were no reports of new linked cases acquired after the public health action was taken.

Legionnaires' disease is difficult to diagnose on clinical grounds. Clinicians caring for patients with communityacquired pneumonia should consider Legionnaires' disease in the differential diagnosis. Infection with $L$. pneumophila serogroup 1 (the serogroup most commonly associated with point-source outbreaks) can be most readily diagnosed using urinary antigen testing. This and other forms of Legionnaires' disease are confirmed by acute and convalescent serology, or identification of the organism in sputum. Cases should be reported to the local public health unit for investigation of the possible source of infection. Staff of the public health unit will carefully question patients about their movements during the incubation period (the 2-10 days before onset of illness) to identify the location of potential sources of infection. It is vital that building managers ensure that any cooling towers are maintained to minimise the risk of contamination with Legionella bacteria.

In April, a further 12 cases of L. pneumophila infection with onset in April were reported in NSW, including five in the South Eastern Sydney Area Health Service. Another case with unconfirmed infection was also reported from South Eastern Sydney. Of these six South Eastern Sydney cases, onset of illness ranged between 12-18 April, ages ranged from 38-71 years, five were smokers, and two reported underlying lung disease.

The South Eastern Sydney Public Health Unit has investigated possible common exposures among these six patients, but none have been found. Only one of the six patients reported visiting the lower part of Oxford Street (10 days before onset of illness).

Clusters of L. pneumophila infection have previously been linked to aerosols from contaminated cooling towers. The South Eastern Sydney Public Health Unit and City of Sydney Council have been searching for evidence of contaminated cooling towers in eastern Sydney. While the majority of cooling towers have been well maintained, two in the Kings Cross area tested positive for Legionella bacteria. Only two of the cases reported visiting that part of the city, and while there is no proof that these towers were the source of their infection, both towers underwent disinfection as a precaution.

\section{Legionella longbeachae infection}

From January to March 2004, four cases of Legionnaires' disease due to Legionella longbeachae infection were reported in the Illawarra and a fifth case was reported in Sydney. The cases were all aged over 60 years and three were men. Two of these individuals died. Four of the five cases reported using potting mix before onset of their illness. L. longbeachae infection has previously been linked to gardening, particularly the use of potting mixes.

In response to this cluster of cases, NSW Health released a media alert to again warn the public of the risk of Legionnaires' disease associated with gardening and the use of potting mix. Reducing exposure to potting mix dust by following manufacturers instructions printed on the potting mix bags is vital in preventing infection from Legionella bacteria. People should avoid breathing in potting mix dust, wear gloves and a mask, and wash their hands immediately after handling potting mix or soil, especially before eating or drinking.

Clusters of legionnaires disease more commonly occur in autumn (see www.health.nsw.gov.au/public-health/phb/ oct01html/epireview.html) and the evidence to date 
suggests that those in the April L. pneumophila cluster are likely to be sporadic and unrelated.

\section{PERTUSSIS IN A NURSERY}

In mid-March, a health care worker who worked at a Sydney hospital was diagnosed with pertussis (whooping cough). The worker reported that flu-like symptoms had begun three weeks before, followed two days later by a cough. The diagnosis of pertussis was confirmed by positive IgA serology. The worker had not received a pertussis-containing vaccine as an adult.

Pertussis is a bacterial infection spread from person-toperson via respiratory droplets. Secondary attack rates in susceptible family members are high. Symptoms begin with a runny nose and a cough that can develop into the classic whooping cough syndrome, with bouts of coughing, followed by an inspiratory whoop, and vomiting. However, symptoms often are not classic, and many adults will only complain of a cough that will not go away. The real danger lies in cases among infants who may require hospital care and will occasionally die from complications such as pneumonia or encephalopathy. Immunisation of children and adults who deal with small children is the mainstay of prevention. The spreading of pertussis can be reduced by identifying and treating infectious cases, and by preventive treatment for their vulnerable close contacts such as newborn babies.

The local public health unit investigated the case to determine whether the infection had spread to other staff or patients in their work area at the hospital. While coughing (but not feeling 'unwell') the worker had worked in a special care nursery and an antenatal clinic.

The public health unit convened an urgent teleconference of a panel involving public health, infectious disease, and paediatric expertise, to determine the best methods for minimising further spread. The panel recommended that parents of the neonates cared for by the worker should be counselled about the risk of pertussis and that the neonates should be offered preventive antibiotics.

Hospital staff contacted parents of the neonates who were identified as having been exposed to the worker and invited them to attend a clinic the next day for counselling and the provision of antibiotics, if indicated. In total, the parents of 20 neonates were counselled. Of these, 16 attended the clinic where the neonates received antibiotics and two were reviewed by other clinicians. The remaining two neonates were exposed more than three weeks before, were asymptomatic on follow-up, and so did not require preventive treatment. No further cases of pertussis were identified.

A review of antenatal patient files indicated the worker did not have significant contact with pregnant women who were of greater than 36 weeks gestation, and thus no further follow-up was required.

While no secondary cases were identified, pertussis is highly infectious, and neonates can have serious complications from the infection. This case highlights the importance of workers in health care and other settings in close contact with young infants being immunised against pertussis, and being aware of the need to have any coughing illness investigated immediately.

\section{SALMONELLATYPHIMURIUM OUTBREAK IN NSW}

Since the beginning of 2004, NSW Health has been investigating a statewide outbreak of Salmonella Typhimurium phage type 12 (STM 12). STM 12 is usually uncommon in NSW with only small numbers of cases reported in the last two years. By the end of April 2004, 126 patients were notified in NSW. Of these, 60 per cent of cases were male and 83 per cent were less than 30 years of age, including 59 per cent under 10 years of age. Public health staff interviewed over 40 of the patients in a search for possible common exposures, but no clear source was identified. Staff from the Hunter OzFoodNet site began a statewide case control study in an attempt to define risk factors for infection in this outbreak.

\section{VIRAL GASTROENTERITIS AT A SPORT CAMP}

A large outbreak of viral gastroenteritis affected over 40 attendees of a hockey camp in the Mid Western Area Health Service. Outbreaks of viral gastroenteritis, often caused by Norovirus infection, are not uncommon in people staying in camps, cruise ships, or aged-care facilities. Reducing person-to-person spread of Norovirus is essential for controlling such outbreaks. People who have symptoms of gastroenteritis should wash their hands thoroughly with soap and running water, and not prepare food or drink or care for patients or others until at least 48 hours after complete recovery. People with symptoms should stay home from work and school.

\section{SEVERE ACUTE RESPIRATORY SYNDROME}

A cluster of nine cases of severe acute respiratory syndrome (SARS) was reported from Northern China in April. The first two cases to become ill ('primary' cases) had worked at a Beijing laboratory where the SARS virus was held. SARS subsequently spread through second and third generations of their contacts. The second generation included two people: a nurse caring for one of the primary cases, and the mother of that primary case. The third generation included five people: three of the nurse's close family members who cared for her while she was ill, and two others who were in the same room as the nurse while she was ill. For more information, see www.who.int/csr/ sars/en/index.html. 
In response, Chinese authorities initiated control measures that included the close monitoring of nearly 1,000 contacts of these cases. At the time of writing (12 May), two incubation periods have now passed since the last of these cases was isolated, and so the further spread of SARS from this cluster is unlikely.

This cluster represents the third and fourth incidents in which laboratory workers have been infected with SARS since the end of the SARS pandemic in mid-2003. The previous infections occurred at laboratories in Singapore and Taiwan. A separate cluster of four unrelated cases was reported from Guangdong province in Southern China in December 2003 and January 2004. The cause of that cluster remains unclear but it is possible that the infections may have been acquired from animals.

These cases highlight the need for clinicians and public health workers to remain vigilant for outbreaks of emerging infectious disease such as SARS. Details on SARS control guidelines can be found at the NSW Health website's Infectious Diseases link, under SARS, at www.health.nsw.gov.au/public-health/alerts/sars/ index.html\#surv.

\section{HIV NOTIFICATIONS IN NSW IN 2003}

The latest analysis of HIV notifications shows that 412 NSW residents were diagnosed with HIV for the first time in 2003 (Table 1). This is a six per cent increase in cases over 2002 (387 diagnoses) and a 22 per cent increase over 2001 (337 diagnoses). Among the 412 cases, 90 per cent were males, 69 per cent reported male-to male sex as a primary risk factor, and 40 per cent were $30-39$ years of age.

As a subset of these notifications, newly-acquired HIV infections (defined here as a negative or indeterminate test within the 12 months prior to the diagnosis, or seroconversion illness at the time of diagnosis) for 2003 showed a 17 per cent increase over 2002, with 144 newlyacquired cases reported in 2003 (compared to 123 cases in 2002 and 98 cases in 2001). Males (95 per cent), maleto-male sexual exposure ( 85 per cent), and people 30-39 years of age (44 per cent) also dominate notifications of newly acquired HIV infection. 


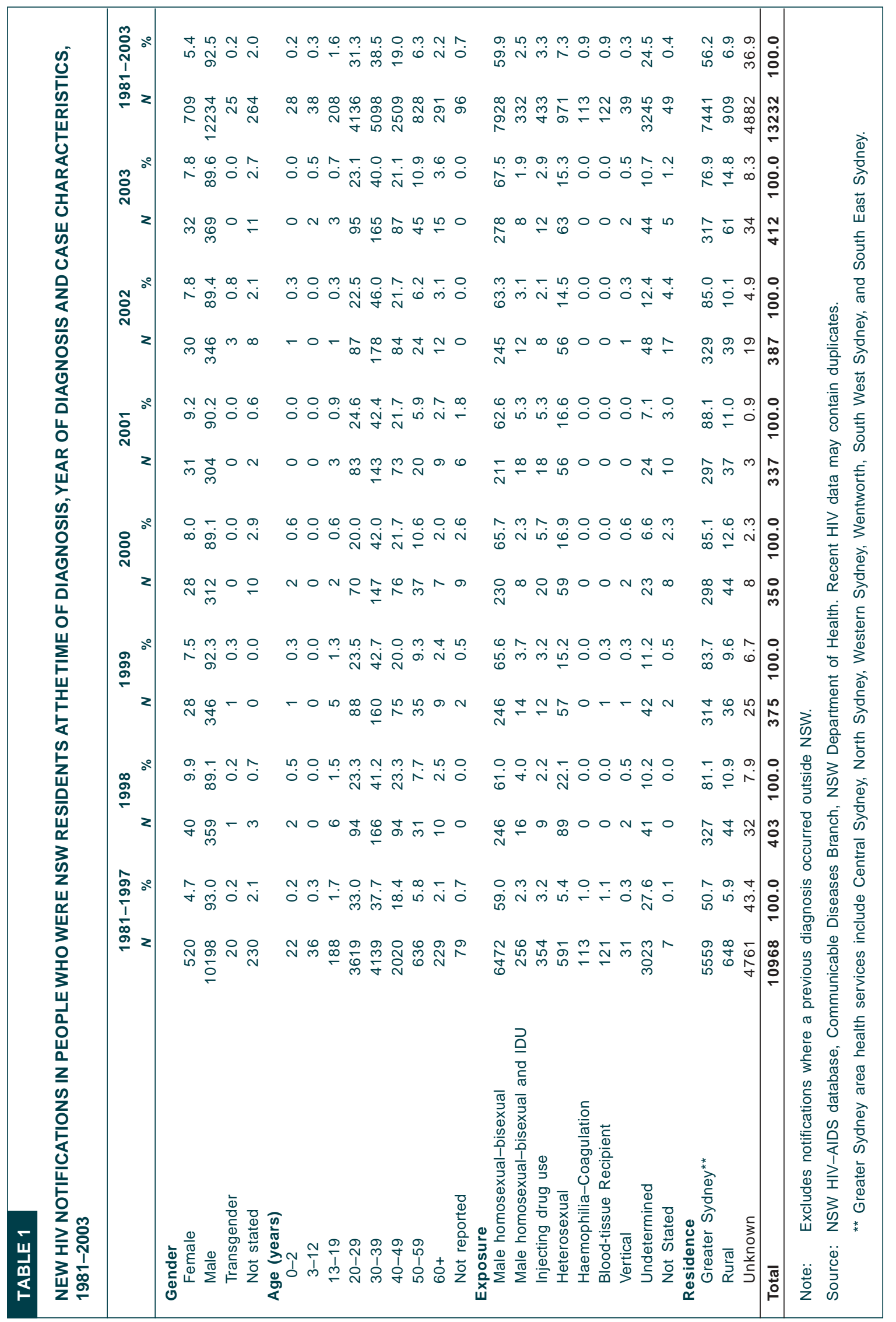




\section{FIGURE 1}

\section{REPORTS OF SELECTED COMMUNICABLE DISEASES, NSW, JANUARY 1998 TO APRIL 2004,}

\section{BY MONTH OF ONSET}

Preliminary data: case counts in recent months may increase because of reporting delays.

Laboratory-confirmed cases only, except for measles, meningococcal disease and pertussis $\mathrm{BFV}=$ Barmah Forest virus infections, RRV $=$ Ross River virus infections

lab+ = laboratory confirmed

Men Gp C and Gp B = meningococcal disease due to serogroup C and serogroup B infection, other/unk = other or unknown serogroups. NB Multiple series in graphs are stacked.

\begin{tabular}{|rc|}
\hline \multicolumn{2}{|c|}{ NSW population } \\
Male & $50 \%$ \\
$<5$ & $7 \%$ \\
$5-24$ & $28 \%$ \\
$25-64$ & $52 \%$ \\
$65+$ & $13 \%$ \\
Rural $^{*}$ & $42 \%$ \\
\hline
\end{tabular}

\section{cases}

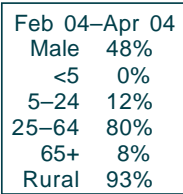

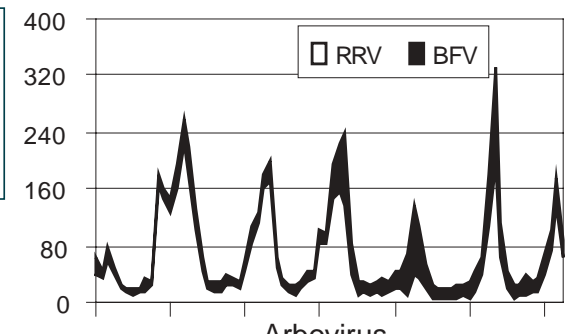

Arbovirus
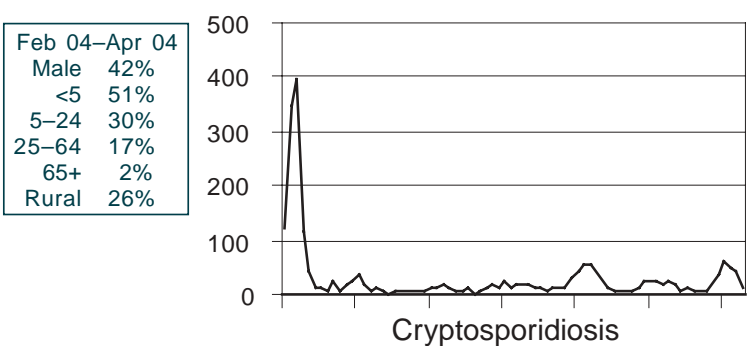

\begin{tabular}{|cc|}
\hline \multicolumn{2}{|c|}{ Feb 04-Apr 04 } \\
Male & $86 \%$ \\
$<5$ & $0 \%$ \\
$5-24$ & $30 \%$ \\
$25-64$ & $69 \%$ \\
$65+$ & $1 \%$ \\
Rural & $22 \%$ \\
\hline
\end{tabular}
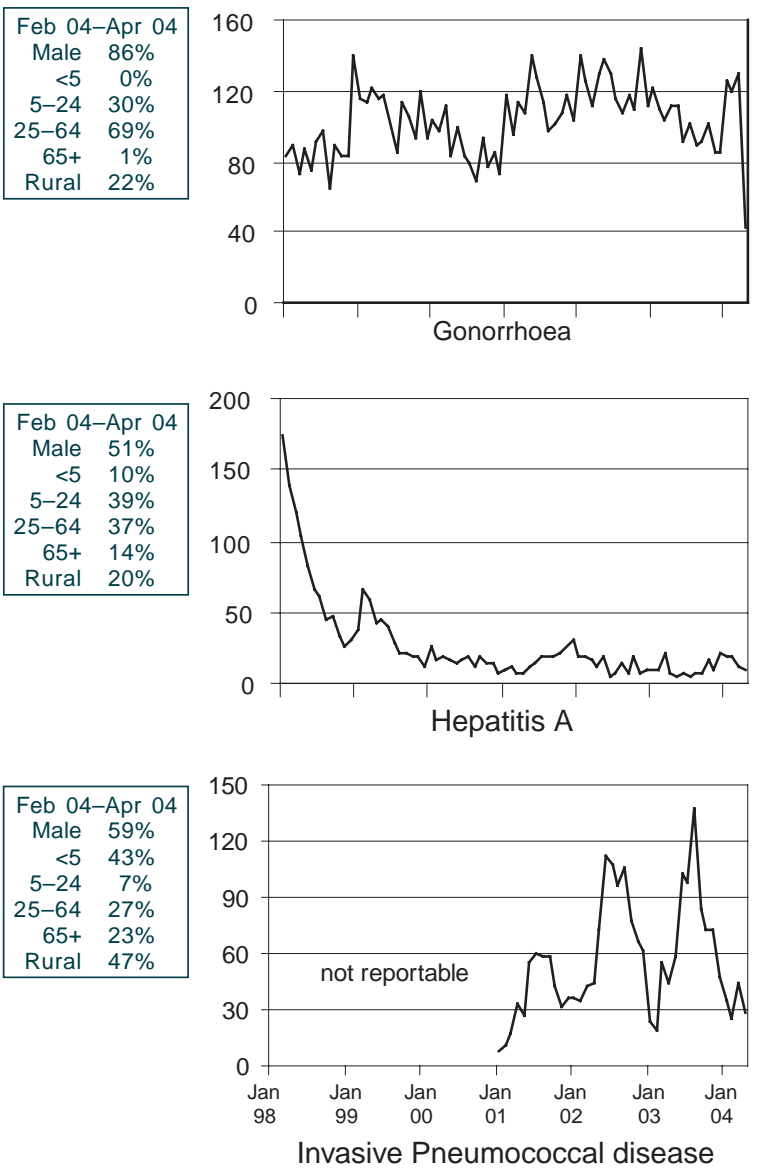

cases

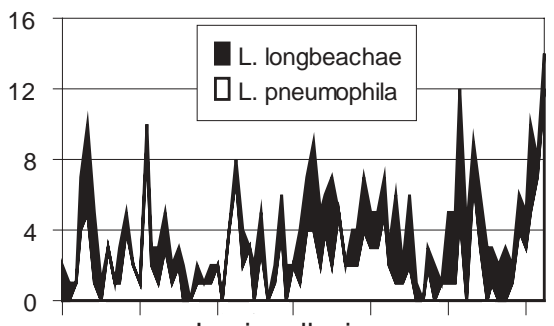

Legionellosis

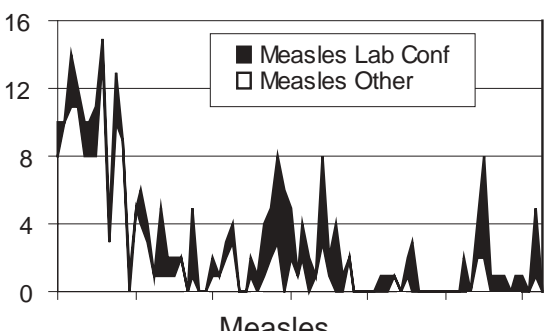

Measles

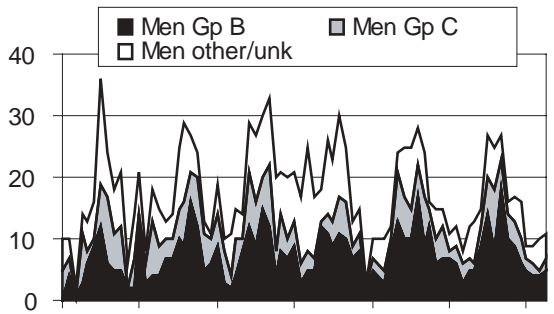

Meningococcal disease
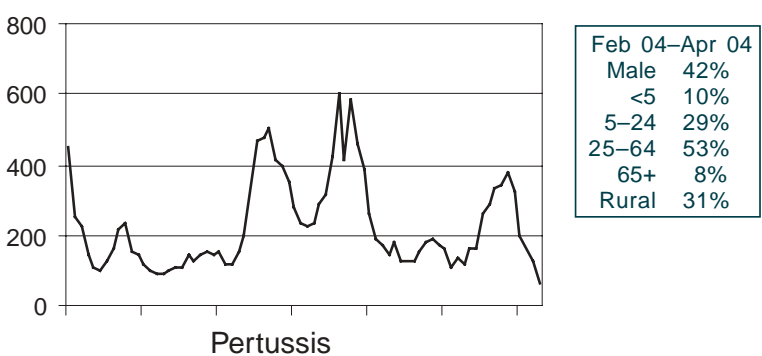

Pertussis

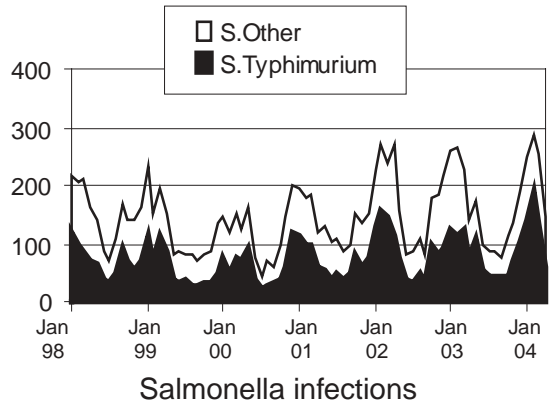

Feb 04-Apr 04 Male $78 \%$ $<5 \%$
$5-24$ $5-24 \quad 3 \%$ $65+38 \%$ Rural $34 \%$
Feb 04-Apr 04 Male $80 \%$ $<5 \quad 20 \%$ 25-64 40\% $65+\quad 0 \%$ Rural 20\% Rural $20 \%$ 5-24 40\%

Rural $47 \%$ 


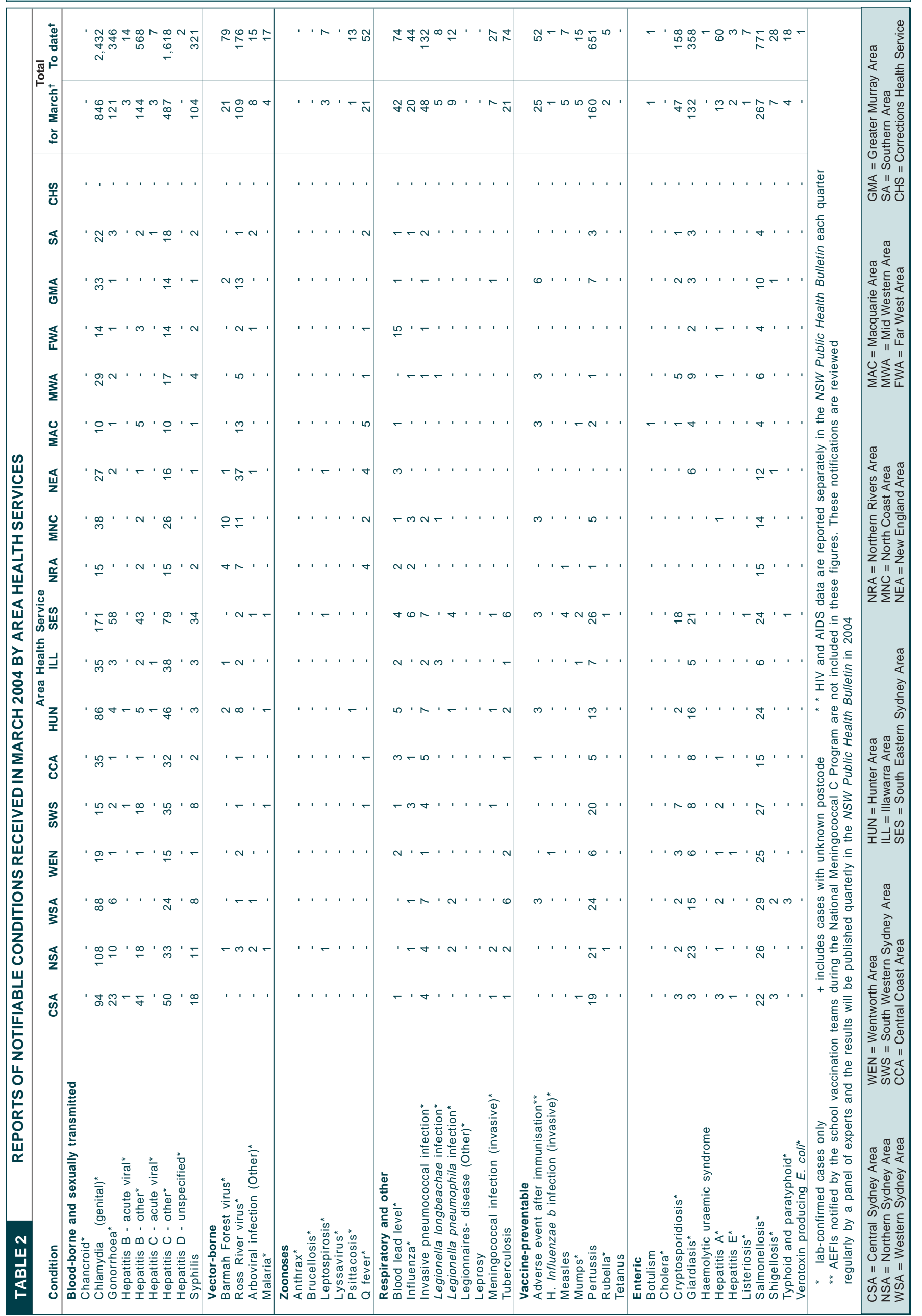




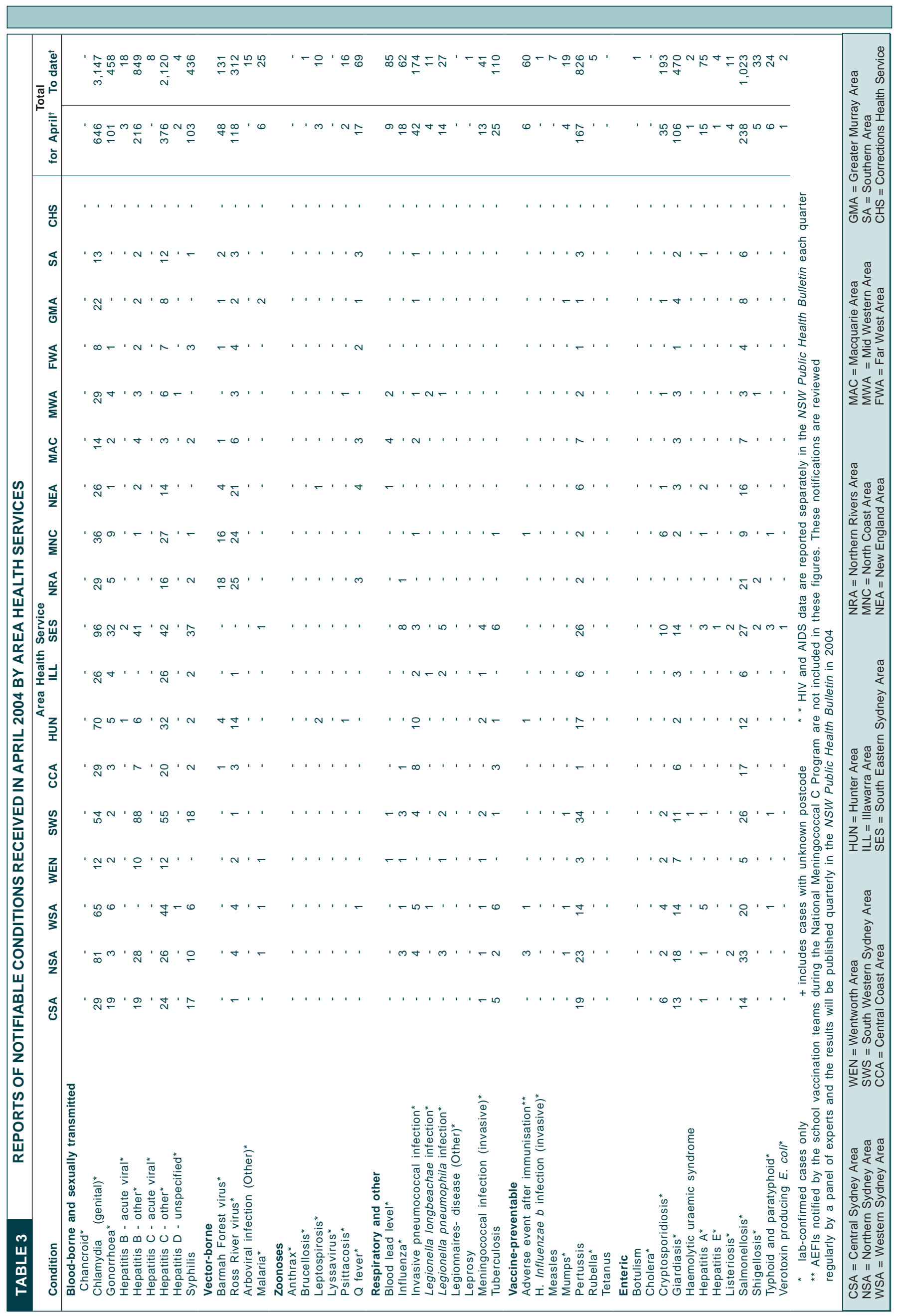

